\title{
PODER REAL AFRICANO. PROPAGANDA Y LEGITIMACIÓN DE JUBA I (CA. 60-46 A. C.) A TRAVÉS DE SUS EMISIONES HELENÍSTICAS
}

\author{
African Real Power. Propaganda and Legitimization of Juba I \\ (ca. 60-46 B.C.) through their Hellenistic Coinage
}

Luis Amela Valverde amelavalverde@gmail.com Grupo CEIPAC. Universitat de Barcelona. España

David Martínez Chico david_ele@live.com Universitat de València. España

Fecha de recepción: 21/12/2018 Fecha de aceptación: 27/02/2019

RESUMEN: Este artículo tiene como objetivo presentar un estado de la cuestión acerca de las acuñaciones monetarias del rey númida Juba I. Históricamente nos encontramos ante la Segunda Guerra Civil Romana mantenida entre Pompeyo y Julio César (49-45 a. C.), donde Juba I apoya al "bando pompeyano». Las monedas de Juba I son producto de dicho acontecimiento y tienen su explicación en el pago de tropas contratadas. Además, las leyendas monetarias se insertan dentro de la propaganda e inciden en la posterior legitimación del trono númida, en manos de Juba I.

Palabras clave: Reino de Numidia; África; acuñaciones; bimetalismo.

ABSTRACT: This paper aims to present a state of the question about the coins struck by Juba I, king of Numidia. Historically we find ourselves before the Second Roman Civil War maintained between Pompey and Julius Caesar (49-45 B.C.), where Juba I supports the "Pompeian faction». The Juba I coins and its relation with the payment of contracted troops is clear. In addition, the monetary legends are propaganda and influence in the subsequent legitimization of the Numidian throne, in hands of Juba I.

Keywords: Kingdom of Numidia; Africa; coinage; bimetallism. 
SUMARIO: 1. Sinopsis histórica. 2. Las monedas de Juba I. 3. Adopciones iconográficas helenísticas. 4. Conclusiones. 5. Catálogo de tipos. 6. Referencias bibliográficas.

\section{SINOPSIS HISTÓRICA}

Juba I [ca. 85-46 a. C.] fue sin duda el último rey legítimo de la Numidia Oriental (ca. 60-46 a. C.), hijo y sucesor de Hiempsal II (88-ca. 60 a. C.), y padre de Juba II, rey de Mauritania (25 a. C.-23 d. C.). Durante su reinado, uno de los cambios de mayor importancia, desde la perspectiva de nuestro estudio, fue respecto a su numerario. De esta forma y por primera vez hace su aparición el bimetalismo plata/bronce, sin obviar el desarrollo de la escala metrológica en bronce; se llevaron a cabo incluso importantes modificaciones, tanto iconográficas como epigráficas; se asistió además al paso de la escritura púnica a la neopúnica y la aparición del latín en las leyendas; e incluso aparecieron representaciones de efigies divinas y retratos reales personificados (Alexandropoulos, 2000, p. 46; 2007, pp. 173 y 185; 2012, p. 227).

En el año 81 a. C. su padre Hiempsal fue repuesto en el trono por el general romano Cn. Pompeyo Magno (cos. 170 a. C.) (App. BCiv. 1, 80. Liv. Per. 89, 7. Plut. Pomp. 12, 6), quien había sido enviado a África por el dictador L. Cornelio Sila (cos. I 88 a. C.), a fin de establecer el orden en la región (Lanzani, 1933, pp. 343-362; Hinard, 1989, pp. 81-88 y Madjoub, 1998, pp. 1321-1328). Este hecho inició el comienzo de una alianza entre los monarcas númidas y Pompeyo. La alianza se hizo aún más fuerte cuando Juba I, todavía príncipe heredero, visitó la ciudad de Roma, y fue insultado por C. Julio César (cos. I 59 a. C.) durante la celebración de un juicio en el que el futuro Dictador defendía a un cliente contra el padre de Juba I (63 a. C.) (Cic. Leg. Agr. 2, 53. Suet. Caes. 71). A lo anterior, hay que sumar la propuesta del tribuno de la plebe C. Escribonio Curión de vender el reino de Numidia (50 a. C.) (Caes. BCiv. 2, 25, 4) (Lacey 1961, pp. 318-329). Ante estos antecedentes, no es de extrañar que Juba I se pusiera de parte de Pompeyo y contra César al estallar la Segunda Guerra Civil Romana (49-45 a. C.).

En agosto del año 49 a. C. César envió a Curión a conquistar a África de manos de los republicanos. Demasiado seguro y teniendo poco aprecio al gobernador de África, P. Accio Varo (vir pr.), Curión no llevó consigo todas las legiones que se le habían confiado para esta campaña. En la batalla de Útica, Curión dirigió a su ejército en un ataque atrevido y difícil, derrotando a las fuerzas de Varo, quien acabó herido en la refriega. Animado por este éxito, Curión atacó lo que él creía que era un destacamento del ejército de Juba I; pero, en realidad, se trataba del grueso de las fuerzas númidas, por lo que, tras un éxito inicial, los soldados de Curión fueron rodeados y aniquilados por Saburra, jefe del ejército de Juba I. Fue la batalla del río Bagradas. Curión, acorralado con los restos de su tropa en una colina, murió en combate. Solo unos pocos hombres lograron escapar a sus barcos. Juba I capturó a varios senadores del bando cesariano; por falta de rescate, terminaron ejecutados 
en Numidia (App. BCiv. 2, 44-46. Caes. BCiv. 2, 23-44. Dio Cass. 41, 41-42) (Offermann, 1977, pp. 351-368; Saylor, 1982, pp. 169-177; Potz, 1998, pp. 293-299; La Penna, 2000, pp. 175-210; Le Bohec, 2004, pp. 1603-1615 y Ott, 2013, pp. 344-415).

En el año 47 a. C. César desembarcó en África a la cabeza de varias legiones para enfrentarse a las fuerzas republicanas, por entonces mandadas por el suegro de Pompeyo, Q. Cecilio Metelo Pío Escipión (cos. 52 a. C.). Juba I tenía originalmente la intención de unirse a este último, pero la invasión de su reino por el oeste, por el aliado de César, el monarca mauritano Boco II y por un aventurero romano, P. Sitio Nucerino, lo obligaron a dejar solo parte de sus fuerzas junto a Metelo Escipión y regresar a Numidia (App. BCiv. 2, 96. BAfr. 24-25. Dio Cass. 43, 3, 2-4).

La victoria cesariana en la batalla de Tapso (46 a. C.), encuentro en que Juba I no participa, y la derrota de sus propias tropas ante Sitio, dejaron en tal situación al monarca númida que, para no caer en manos de César, termina haciendo un pacto suicida con su amigo M. Petreyo (pr. ca. 64 a. C.), participando en una pelea cara a cara. La idea era que uno de ellos tendría una muerte honorable. Las fuentes difieren (App. BCiv. 2, 100. BAfr. 94. Dio Cass. 43, 8, 4. Eutrop. 6, 23, 3. Flor. 4, 2, 69. Liv. Per. $114,4$. Oros. $6,16,3)$, pero es probable que Petreyo matara a Juba I, y luego se suicidara con la ayuda de un esclavo. Su hijo y sucesor, Juba II, será criado en Roma en un cautiverio dorado por Octavia, la hermana del futuro emperador Augusto (27 a. C.-14 d. C.) (McDermott, 1969, pp. 858-862; Cowan, 2008, pp. 24-29; Gozalbes Cravioto, 2016, pp. 65-82; Porte, 2017, pp. 61-81 y McDonnell-Staff, 2018, pp. 28-37).

Como se sabe, la clientela pompeyana en Numidia estaba muy consolidada desde hacía algunos años. No obstante, la gens Pompeia en África se vinculaba más bien a la propia e imparable carrera del propio Pompeyo. Un hecho trascendental fue la guerra contra los marianistas (81 a. C.) y la posterior restitución de Hiempsal Il en el trono, el padre de Juba I, lo que produciría en el romano una innegable fama entre todos los reinos indígenas africanos (Amela Valverde, 2000, pp. 254-255, y 2002 para la clientela hispana, interconectada con la africana).

\section{LAS MONEDAS DE JUBA I}

La amonedación del bando senatorial durante la Segunda Guerra Civil Romana (49-45 a. C.) contrasta de muchas maneras con la efectuada por César. Para M. H. Crawford, quizás lo más sorprendente, incluso cuando Pompeyo todavía estaba con vida, fue su moneda militar y que, a diferencia de la de César, fue producida por un gran número de lugartenientes, en parte con tipos familiares o privados (Crawford, 1974, p. 737). Esto realmente no tiene por qué llamar la atención, debido a que, en realidad, César no luchaba contra Pompeyo, sino contra el Senado, por lo que con propiedad podría hablarse de bando senatorial y no de bando (y menos partido) pompeyano. 
Después de la muerte de Pompeyo, sus seguidores se refugiaron en África, donde produjeron áureos, denarios y quinarios: Metelo Escipión acuñó tanto solo como junto a dos legados diferentes (RRC 459-461); M. Porcio Catón (pr. 54 a. C.) abandonó finalmente sus principios y emitió moneda en su propio nombre ( $R R C$ 462), aunque copiando los tipos de M. Porcio Catón, monetario en el año 89 a. C. (RRC 343) (Crawford 1974, p. 738).

Esta amonedación fue aumentada por una gran producción de denarios $(R P C)$ 717) (cat. mons. no 1-2) (Crawford, 1985, p. 246-247) por mano de Juba I, a la que D. R. Sear añade los quinarios (RPC I 718-719) (cat. mons. no 3-4), los sestercios (RPC। 720) (cat. mons. no 5) y los bronces mucho más raros efectuados por este monarca que, en su mayoría, llevan el nombre y el retrato de este príncipe, y presumiblemente habrían sido acuñadas en su capital, Zama (BAfr. 91, 1) (Sear, 1998, p. 31), que habría obtenido tal estatuto tras la partición de los territorios a la muerte de Gauda (105-88 a. C.), entre sus dos hijos (Coltelloni-Trannoy, 2003, p. 3917).

Más bien, solo las monedas de plata serían acuñadas por Juba I durante este conflicto (Burnett, 1987, p. 176; Burnett, Amandry y Ripollès, 1992, p. 190; Woytek, 2003, pp. 240-241; Kormikiari, 2007, pp. 259 y 284; 2014, p. 23). Hemos de decir que Chaves Tristán (2005, p. 222) considera que Juba I emitiría solo los denarios y los quinarios durante la guerra contra César, aunque es de suponer que los bronces serían anteriores y de carácter más bien local (Bertrandy 1976-1978, p. 17; Burnett, Amandry y Ripollès, 1992, p. 182), ya que quinarios y sestercios fueron reintroducidos por César en el año 48 a. C. (Burnett, 1987, p. 176; Woytek, 2003, p. 241 y Günther, 2018, p. 7); los primeros denarios aparecen en tesoros fechados en el año 46 a. C. (RRCH 383-384), lo que confirma que estos fueron acuñados a finales de su reinado (Burnett, 1987, p. 176), en el año 47/46 a. C., con objeto de apoyar la causa senatorial (Maritz, 2001, p. 114; Kormikiari, 2007, pp. 259, 284 y 288; Kormikiari, 2014, p. 23; Falomir Pastor, 2013, p. 41 y Estarán Tolosa, 2016, p. 486) y con el evidente objetivo de hacer frente a los gastos derivados del conflicto bélico, entre ellos, por supuesto, el pago de la soldada.

A partir de la comparación efectuada a través de los distintos ocultamientos, A. $M$. Burnett considera que la producción de denarios por parte de Juba I fue mayor que la de Catón, pero más pequeña que la de Metelo Escipión (Burnett 1987, p. 177). Por el contrario, Alexandropoulos (2000, p. 47) defiende que las emisiones de Catón y de Juba I son más numerosas que las de Metelo Escipión, que parecen ser más raras.

Actualmente sabemos que los denarios fueron producidos en grandes cantidades y se encuentran conjuntamente con los denarios romanos en tesoros de época republicana e imperial, los quinarios y los sestercios son muy raros (Bertrandy, 1976-1978, p. 17; Burnett, 1987, pp. 176-177; Burnett, Amandry y Ripollès, 1992, p. 190; Alexandropoulos, 2000, p. 47; 2005, pp. 206 y 2007, p. 177; Woytek, 2003, p. 241), al revés que el numerario de Catón, donde predominan los quinarios sobre 
los denarios (Alexandropoulos, 2000, p. 47 y 2007, p. 177). En efecto, la abundancia de este valor corresponde al predominio del denario como la pieza de plata por excelencia y en el occidente mediterráneo.

Hemos de destacar la sugerencia de Loscheider (1997, p. 23) pues según este autor la imaginería de los quinarios del caudillo Trévero Arda (DT 603-605) procede del quinario de Juba I (RPCI 718) (cat. mons. no 3). En cualquier caso, la intercambiabilidad/convertibilidad de los ejemplares acuñados por Juba I con las monedas romanas puede observarse en su amplia circulación y en tesoros de finales de la República e inicios del Imperio (vid. RRCH 383, 384, 409, 421, 432, 433, 437, 440, 442, 443, 460, 465, 471. 475, 478. 479, 488, 491, 493, 506, 508, 511, 516, 528, 531, 543 y 546) (Burnett, Amandry y Ripollès, 1992, p. 182, Kormikiari, 2007, pp. 284 y 2014, p. 23, Günther, 2018, p. 8). Destacan ocultaciones y hallazgos esporádicos en Hungría, Croacia, Bosnia-Herzegovina, Albania (Mirnik, 1987, p. 376, Xhyheri y Muçaj, 2014, p. 83), Grecia (Sidiropoulos, 2011, p. 1132), Italia (Ranucci, 2007, p. 151), Sicilia (Walthall, 2013, p. 175), Francia (Fischer, 1978, pp. 117-123; Burnett, Amandry y Ripollès, 1992, p. 190; Coltelloni-Trannoy, 2003, p. 3920 y Falomir Pastor, 2013, p. 41) y Alemania (Günther, 2018, p. 5), no contemplados en la lista de $R R C H$.

De hecho, desde un punto de vista conceptual, las emisiones de plata de Juba I son ciertamente romanas. Sus denominaciones, una leyenda latina en el anverso, la utilización del retrato de Juba I a dcha. (en vez de a la izq., como era tradicional) y la presencia de una victoria en los quinarios muestran la influencia romana en esta amonedación (Bertrandy, 1976-1978, pp. 12-18; Woytek, 2003, p. 241; Alexandropoulos, 2005, pp. 207 y 2007, p. 176; Estarán Tolosa, 2016, p. 485), aunque no es menos cierto la existencia de un aporte helenístico egipcio.

Por ello, no es de extrañar que se haya considerado que las series de plata del monarca númida habrían sido producidas por artesanos romanos, no en Zama, sino en la provincia de África, en Útica (Mazard, 1955, p. 49; Burnett, Amandry y RipoIlès, 1992; p. 190; Alexandropoulos, 2000, pp. 46-47; 2005, pp. 205 y 207; 2007, pp. 174 y 2012, p. 228; Kormikiari, 2007, p. 259; Estarán Tolosa, 2012, pp. 353 y 2016, p. 484; Falomir Pastor, 2013, p. 41; Xhyheri y Muçaj, 2014, p. 83). Pero esta teoría está basada sobre la consideración que todas las emisiones senatoriales del Norte de África se produjeron en dicha ceca, lo cual no es cierto (Woytek, 2003, p. 244 y Günther, 2018, p. 7).

Para B. Woytek, la existencia de un bronce de Juba I (MAA 34) (cat. mons. no 7) en la que figura un templo que es casi similar al que figura en los denarios (RPC I 717) (cat. mons. no 1-2), sugiere que las emisiones del monarca númida se efectuaron en su propio territorio. El patrón metrológico, el empleo de artesanos romanos, así como el uso de leyendas latinas podrían haber tenido únicamente razones prácticas o político-ideológicas, y son independientes al lugar de acuñación. En cualquier caso, el citado investigador deja abierta la cuestión de la localización del taller (Woytek, 2003, p. 244). 
Por su parte, J. Alexandropoulos divide la producción argéntea de Juba I en dos cecas, Útica (RPCI 717-718) (cat. mons. no 1-3) y Cirta (RPCI 719-720) (cat. mons. no 4-5) (Alexandropoulos, 2007, pp. 401-402). Como en la primera sede no parece ser posible que se ubicara el taller para producir moneda para el rey númida, quizás Cirta (act. Constantina, wil. Constantina, Argelia), "la ciudad más rica del reino» (BAfr. 26, 2), podría ser la candidata mejor posicionada. Sobre Zama, su «residencia», en donde había depositado todo su dinero y cosas de valor (BAfr. 91, 1), le cerró las puertas tras conocer su derrota en Thapsus (BAfr. 91,2) y se pasó a manos de César (BAfr. 92, 1-2); por tanto, no parece haber sido precisamente una plaza de confianza para el establecimiento del taller.

En un principio se ha considerado que Cirta se encontraría en la Numidia occidental, según G. Camps, cuyo monarca no era Juba I, sino Masinisa II (81?-46 a. C.), posiblemente vasallo del primero y que no acuñó moneda (Camps, 1981, pp. 308,311 y 77). Este mismo autor, más tarde, cambia de opinión y considera que se encontraría en la Numidia Oriental, que habría conservado el viejo nombre de Numidia (Coltelloni-Trannoy, 2003, p. 3915). De esta forma, podemos considerar en principio que fue en Cirta donde estuvo establecida la ceca de moneda de Juba I, durante el conflicto civil romano. En el mismo planteamiento se sitúan también Alexandropoulos (1992, pp. 143 y 2007, p. 402) y Estarán Tolosa (2012, p. 353, n. 38), al considerar que las amonedaciones broncíneas de Juba I fueron acuñadas en la ceca de Cirta, mientras que Günther (2018, p. 6) duda entre Cirta o Zama.

\section{ADOPCIONES ICONOGRÁFICAS HELENÍSTICAS}

En cuanto a iconografía y leyendas, comencemos por las series en plata, que hacen su reaparición en el numerario númida desde la época de Sifax (ca. 215-ca. 203 a. C.) (Alexandropoulos, 2000, p. 173). La primera diferencia es que tanto el denario ( $R P C I$ 717) (cat. mons. $\mathrm{n}$ - 1-2) como uno de los dos tipos de quinarios ( $R P C$ I 718) (cat. mons. $n$ o 3) presentan en su anverso la leyenda latina REX IVBA mientras que en el reverso la neopúnica YWB'Y HMMLKT (Kormikiari, 2007, p. 284 y Günther, 2018, p. 7); a señalar que el valor y la leyenda del anverso son de carácter romano, pero no su iconografía (Günther, 2018, p. 7). Por primera vez en África, la titulatura aparece en el reverso como en las monedas romanas (Coltellony-Trannoy, 2003, p. 3920). No solo eso, sino que también es la primera vez que se utiliza el latín en la amonedación real númida (Alexandropoulos, 2007, p. 176 y Kormikiari, 2007, p. 284).

Por el contrario, el otro tipo de quinario (RPC I 719) (cat. mons. $\mathrm{n}$ o 4) así como el sestercio ( $R P C I 720$ ) (cat. mons. $n$ ㅇ 5) no presentan leyenda alguna, lo que ha motivado que se haya considerado que el primer grupo se hubiera producido en un taller diferente al segundo (como hace J. Alexandropoulos) o que incluso se 
hubieran efectuado en un momento histórico diferente, algo difícil de considerar debido a que las fracciones del denario habían estado mucho tiempo sin acuñar hasta el estallido de la segunda guerra civil romana (49-45 a. C.), como ya hemos señalado.

Desde el punto de vista iconográfico, tanto el denario (RPC I 717) (cat. mons. no 1-2) como el quinario anepigráfico (RPCI 719) (cat. mons. $n$ ㅇ 4) presentan un retrato del rey Juba I; algo nada novedoso, sino todo lo contrario, ya que los diversos monarcas aparecen representados en el anverso de sus monedas (MAA 1-25). Pero hay una diferencia: el retrato está hacia la derecha y no hacia la izquierda, como era tradicional en las monedas norteafricanas. Asimismo, la imagen del monarca no aparece en las emisiones de bronce, sino en algunas de plata (Alexandropoulos, 2007, p. 173). Al igual que en los denarios (cat. mons. no 1-2), en la moneda de bronce de Juba I (MAA 34) (cat. mons. no 7) ${ }^{1}$ en el reverso también figura el templo octástilo, mientras que en el quinario aparece el caballo a galope, elemento tradicional númida (así como cartaginés) (Günther, 2018, p. 7).

A su vez, en el quinario con leyendas figura en el anverso un busto de la Victoria (RPC I 718) (cat. mons. no 3), un elemento característico de los quinarios romanos de este tiempo, mientras que el sestercio presenta una imagen clásica de África (RPCI 720) (cat. mons. no 5), elemento de origen helenístico. En cuanto al reverso del quinario se figura de nuevo un caballo a galope, al igual que en el quinario con leyendas (lo que podría indicar que ambos tipos pudieron fabricarse en una misma ceca), mientras que en el reverso aparece un león, perteneciente a la imaginería tradicional númida (Mazard, 1955, p. 50).

Los bronces (cuatro valores), en contraste con las monedas de plata, presentan en su reverso la leyenda neopúnica SYWB'Y HMMLKT, es decir, que mencionan la naturaleza real de Juba I. Estas piezas presentan elementos iconográficos que ya se encuentran presentes en las emisiones de plata: templo octástilo (MAA 34) (cat. mons. no 7), busto de África y león (MAA 36) (cat. mons. no 9) y misma disposición que el sestercio ( $R P C \mid 720$ ) (cat. mons. no 5). Por el contrario, aparece la figura de Amón en el anverso y un elefante en el reverso (MAA 33 y 35) (cat. mons. no 6 y 8), así como un edificio con cinco columnas (MAA 34) (cat. mons. no 7)².

${ }_{1}^{1}$ Para Mazard (1955, p. 50) es a partir de Juba I cuando se representa el santuario púnico como templo greco-romano clásico, aunque Xhyheri y Muçaj $(2014$, p. 84) indican que el techo plano es de estilo púnico, por lo que estaría probablemente dedicado a Baal.

${ }^{2}$ Mazard (1955, p. 51) indica que Falbe, Lindberg y Müller (1862, pp. 43-44), además de Gsell (1927, p. 160), consideraron que se trataba de una representación de un palacio real en Cirta o Zama. Por su parte, Coltellony-Trannoy (2003, p. 3920) piensa que pueden tratarse de monumentos de Zama, embellecida y fortificada por Juba I (Vitruv. 8, 3), o de Cirta, embellecida por los arquitectos de Micipsa. 
Puede pues observarse que en los motivos representados en la amonedación de Juba I existe una iconografía mixta, de motivos indígenas (caballo, león ${ }^{3}$, elefante) ${ }^{4}$ y greco-romanos (templo, edificio ${ }^{5}$, Victoria, Amón, África) (Alexandropoulos, 2000, pp. 46-47; Coltellony-Trannoy, 2003, p. 3920; Kormikiari, 2007, p. 284 y 2014, p. 23; Günther, 2018, p. 7) ${ }^{6}$. En cierta forma, no parece que pueda existir contradicción alguna en encontrar motivos y similitudes de carácter individual en las monedas acuñadas por la facción optimate en África (RRC 459-462), especialmente la cabeza de Júpiter que aparece en una emisión de Metelo Escipión (RRC 459/1), conjuntamente con un elefante. Pudiera tratarse de una estrategia coordinada por los enemigos de César, lo que no tiene que significar que existiera un único taller para todas estas emisiones (Günther, 2018, p. 7).

La figura de África tocada con piel de elefante se interpreta en función de muchas referencias, aunque claramente nos encontramos ante una inspiración helenística. Esta imaginería aparece por primera vez en unas monedas cuyos orígenes se remontan a la efigie del deificado Alejandro Magno de Macedonia (336-323 a. C.) y que el sátrapa (luego rey) Ptolomeo de Egipto (323-283/282 a. C.) colocó en su emisión inicial de tetradracmas de plata, poco después del año 323 a. C. (Salcedo Garcés, 1996, pp. 128-130, Coltelloni-Trannoy, 1999, p. 67, Sear, 1998, p. 33 y Amisano, 2014, p. 180); pero también es una manifestación del derecho del monarca sobre Numidia, ya que en el tocado de la figura del anverso (MAA 36) (cat. mons. no 9) la trompa está erguida y se relaciona con las primeras emisiones de origen africano de

${ }^{3}$ Según Mazard (1955, p. 51) el león puede interpretarse como emblema nacional, sin que se descarte que pueda estar relacionado en Fenicia con la diosa Astarté, si bien puede ser un atributo de su parecido cartaginés Tanit, como alusión al carácter guerrero de dicha deidad, ya que a veces se representa a esta divinidad con cabeza de león.

${ }^{4}$ Falbe, Lindberg y Müller (1862, p. 44) señalan que estos tres animales, representados siempre en el reverso de la amonedación de Juba I, son los característicos de Numidia.

${ }^{5}$ Trell $(1976$, p. 13 y 1988, p. 53) considera que se trata de un santuario con tres altares. Coltellony-Trannoy $(2003$, p. 3920) sugiere que la representación de monumentos en las monedas es de carácter romano, que aparece (más bien se difunde) durante el siglo i a. C., como, por ejemplo, la basilica Aemilia (RRC 419/3) o la Villa Publica (RRC 429/2), donde pudo haber estado alojado Juba I durante su estancia en Roma. Precisamente, Bertrandy (1980, p. 14) piensa que se tratan de construcciones de edificios públicos de carácter civil, tomados como modelo para las mencionadas emisiones. Pero tampoco debemos ignorar, como bien señala Pera (1998, p. 131), que Juba I simplemente se dedicara a reproducir en sus monedas todos esos elementos arquitectónicos y que bien pudo haber visto durante su estancia en Roma.

${ }^{6}$ Es extraño que Günther (2018, p. 7) liste el busto de África entre los elementos de carácter indígena, ya que, como indica Mazard (1955, p. 51), su aparición se da en la numismática ptolemaica, y figura así mismo en la amonedación romana, como en el célebre áureo de Pompeyo (RRC 402/1). Sobre ello vid. Amela Valverde, 2001, pp. 5-13; 2010, pp. 205-216 y 2013, pp. 11-14; Campana, 2001, pp. 20-24; Mesiano, 2011, pp. 93-113; Woytek, 2015, pp. 403-426; Kopij, 2016, pp. 109-127 y Marshall, 2016, pp. 107-133. 
este tipo (Mazard, 1955, pp. 94-95 y 97-98) ${ }^{7}$, pero que M. Coltelloni-Trannoy atribuye, de manera errónea pero involuntaria, al seguir la opinión de Mazard (1955, pp. 53-54), al usurpador Hiarbas, quien ocupó el trono de Hiempsal II. Por otro lado, en el anverso del sestercio argénteo ( $R P C I$ I20) (cat. mons. $\mathrm{n}$ - 5) se opta por un tocado con trompa bajada entre las defensas, aunque se trata del mismo motivo -coetáneo- reproducido en el denario de Metelo Escipión (RRC 461/1), el aliado de Juba I, acuñado también en África (Coltelloni-Trannoy, 1999, p. 70 y 2003, p. 3920).

La figura del león en los bronces (MAA 36) (cat. mons. no 9) podemos interpretarlo como un blasón del poder real. Realmente se trata de una manifestación de la pujanza del gran dios africano, asociado al poder y a Baal Amón (aunque más bien se trataría de Zeus Amón). El león del Atlas es, pues, un símbolo tanto real como nacional, que representa tanto a Numidia como a su gobernante, una «personificación» del reino, que podría implicar una fuerte asociación personal entre Juba I y este felino (Coltelloni-Trannoy, 1999, p. 91).

En este sentido, el elefante es un animal asociado a la potencia benéfica de la deidad tutelar, en este caso Amón, que se encuentra en el anverso de dos tipos de bronces de Juba I, mientras que en el reverso de ambas figura el mencionado paquidermo (MAA 33 y 35) (cat. mons. no 6 y 8) (Coltelloni-Trannoy, 1999, p. 91).

Los denarios presentan en su anverso la efigie del monarca (RPC I 717) (cat. mons. no1-2), fiel a la moda africana que menciona Estrabón (Str. 17, 7, 2. Cf. Sil. Ital. Pun. 3,284$)$ y comparable con las descripciones que han llegado hasta nosotros sobre Juba I (adulcescens bene capillatus [Cic. De Leg. agr. 2, 22]), en los que podemos destacar que lleva bigote y barba, así como una diadema, insignia de la monarquía helenística, que se complementa con un cetro y la leyenda REX IVBA (Falbe, Lindberg y Müller 1862: 43, Coltelloni-Trannoy 2003: 3921).

Juba I fue nombrado "rey» por el senado optimate reunido en Tesalónica (Dio Cass. $41,42,7)$ tras su victoria sobre Curión, a lo que habría que añadir la fórmula típica de "amigo y aliado» (Coltelloni-Trannoy, 2003, pp. 3919 y 3923), que ya se le intentó otorgar por parte del Senado a principios del año 49 a. C., y que no llegó a buen término (Caes. BCiv. 1, 6, 4). Estos títulos reconocían a Juba I su preeminencia local a los ojos de los romanos, que muestra la verdadera posición del monarca númida en su reino: extraordinariamente frágil ya que va ligada a la protección romana.

El título rex que aparece en el denario (RPCI 717) (cat. mons. $\mathrm{n}$ - 1-2) y en el quinario con leyendas (RPC I 718) (cat. mons. $\mathrm{n}$ - 3) de Juba I traduce de manera imperfecta título africanos: mastan («el protector»: MSTNS en las monedas MAA

7 Únicamente hemos encontrado en la obra de J. Alexandropoulos (MAA) el equivalente a Mazard 96 = MAA 37, que correspondería según Alexandropoulos (2007, p. 403) a un bronce perteneciente a taller desconocido, ubicado en Numidia occidental o Mauritania y que habría emitido moneda durante los años ca. 60-50 a. C. (?). 
40-41 [que M. Coletellonny-Tranoy atribuye al monarca mauritano Soso]), gellid o agellid* (GLD o GLDT en un epígrafe bilingüe de Dougga) o en (neo)púnico mamleket, "realeza», o mejor "persona real», "señor del reino» (HMMLKT en las monedas de Juba I [RPC I 717-718 y MAA 33-36] [cat. mons. no 1-3 y 6-9]). Sin duda, una clara declaración política (Pera, 1998, p. 1330, Coltelloni-Trannoy, 2003, pp. 3919-3920, Kormikiari, 2005, p. 350 y Alexandropoulos, 2007, p. 177).

La aparición de la efigie, diademada, del monarca en la moneda de más valor y trascendencia, el denario, con una titulación soberana completo, REX IVBA en latín y YWB'Y HMMLKT en neopúnico, acompañada de un cetro y un manto ( $R P C I$ 717) (cat. mons. $\mathrm{n}$ - 1-2), tenía de manera presumible como fin legitimar un soberano reconocido de mala gana por sus aliados optimates y para quien una victoria cesariana en el conflicto significaba pura y simplemente la anexión inmediata de su reino (Alexandropoulos, 2012, p. 228). Se trata de una imagen política, que contrasta en cierta manera con la de carácter militar que transmite la Victoria en los quinarios ( $R P C$ I 718) (cat. mons. $\mathrm{n}$ - 3) o la representación de África en los sestercios ( $R P C$ I 720) (cat. mons. $\mathrm{n}$ o 5), o incluso la del bronce que empareja a Amón con un elefante (MAA 35) (cat. mons. $\mathrm{n}$ - 8). La idea de "un reino en pie de guerra» prevalece (Alexandropoulos, 2012, p. 229).

Por el contrario, las monedas de bronce muestran por primera vez que el nombre y la titulatura del monarca no van ligados a su retrato, sino, tanto en el anverso como en el reverso, a diferentes motivos, como divinidades (Amón [MAA 33 y 35]), alegorías (África [MAA 36]), o animales (león [MAA 36], elefante [MAA 34-35]).

Se trata, pues, del primer intento de representación monetaria no solo del monarca sino de su reino con sus dioses tutelares, y en este sentido puede decirse, en palabras de J. Alexandropoulos, que es «una especie de invención iconográfica monetaria de la Numidia por los propios númidas». Por tanto, la reforma del numerario real númida efectuada por Juba I pretendía reafirmar la autoridad real en periodo de crisis, pero a la vez propone así mismo la primera evocación del reino en tanto que tal, al romper con el paisaje monótono de la representación del soberano en la amonedación númida (Alexandropoulos 2012, pp. 229 y 230).

\section{CONCLUSIONES}

Un juicio que compartimos es el de M. Coltelloni-Trannoy, al considerar que la amonedación de Juba I no fue una simple imitación de los modelos romanos, sino la adopción de técnicas y tipos que muestran el poder de Juba I frente al de sus aliados romanos, ya que pretende ser tratado de igual a igual. La mera integración de su numerario en el sistema romano es un indicativo fuertemente sólido de la rápida evolución del mundo africano hacia la romanización (Coltelloni-Trannoy, 2003, pp. 3920-21-22). 
Por nuestra parte, Juba I fue totalmente consciente del uso propagandístico que los romanos hacían de la moneda. En efecto, la legitimación a través del soporte numismático era una herramienta que para Juba I no iba a pasar desapercibida. Recientemente otros autores modernos (Falomir Pastor, 2013, p. 42) también han recalcado la hipótesis de que las amonedaciones tardías del rey Juba I surgieron durante la guerra civil mantenida frente a Julio César, apoyando al bando pompeyano. Por tanto, la principal causa de estas emisiones númidas, tanto en plata como en bronce, se encuentra en el pago de las innumerables tropas que eran contratadas para dicha causa. De esta forma, Juba I adopta la metrología romana con tal de que el numerario fuera aceptado en todo el Mediterráneo. Así podemos encontrar tanto denarios, quinarios y sestercios argénteos (cat. mons. no 1-5), como piezas de metrología nuevamente imperial, caso del sestercio, dupondio y as (cat. mons. $\mathrm{n}$ 은 6-9).

A nivel general, las cecas que supuestamente corrieron a cargo de todas las emisiones de Juba I debieron ser, en una primera fase, Útica, con emisiones argénteas de denarios y quinarios (cat. mons. $n=1-3$ ), y, en una segunda fase, aunque a cargo del taller de Cirta, otros quinarios y sestercios, además de los sucesivos múltiplos emitidos en bronce (cat. mons. no 4-9) (Alexandropoulos, 2007, pp. 174-186).

De esta forma, la «romanización» de la amonedación africana (tanto en la metrología como en ciertos elementos iconográficos), que aparece a finales del reinado de Juba I, ante la necesidad perentoria de dinero para hacer frente a las fuerzas militares del bando cesariano, no es un signo de debilidad, como pareciera a primera vista, sino todo lo contrario, de afirmación política del monarca en el seno del mundo romano (Alexandropoulos, 2007, p. 48). ¡Qué mejor forma de transmitir el mensaje de que Juba I es el monarca de Numidia que en la misma lengua y escritura de la potencia dominante en la cuenca del Mediterráneo!

Las monedas de Juba I se sitúan en una larga tradición, procedente de la época del monarca númida Masinisa I (202-148 a. C.), mostrando una evolución del poder real en África hacia el modelo político de los estados helenísticos (como el de Roma). Juba I acuña moneda para afirmar la identidad y la autoridad de su poder, tanto frente a las fuerzas internas (de su reino) como a las externas (es decir, los romanos). 


\section{CATÁLOGO DE TIPOS}
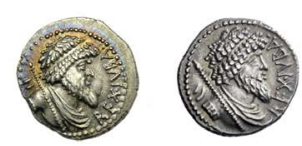

2
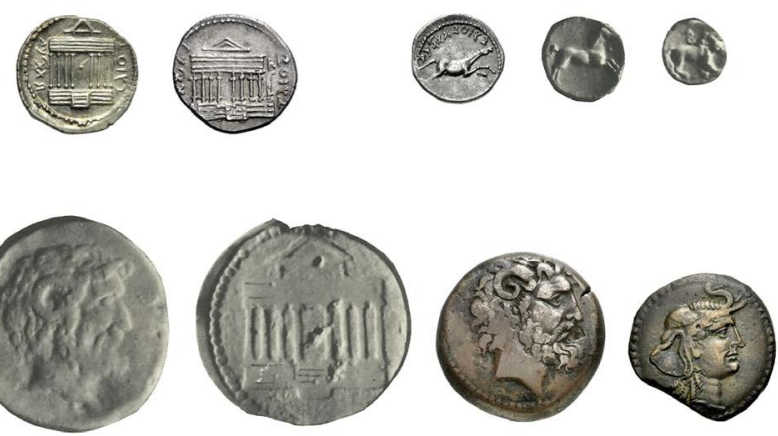

6
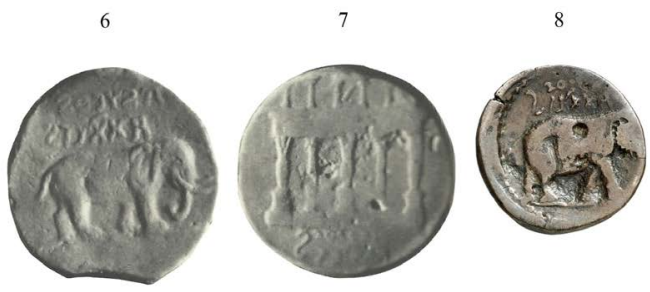
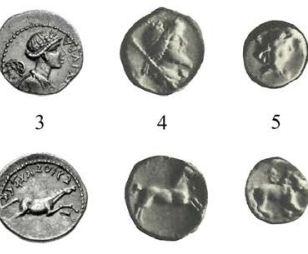

5

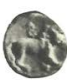

\section{TALLER DE ÚTICA}

1. Denario de Juba I (Mazard 1955: no 84; Alexandropoulos 2007: no 29B) (3.78 g; $19 \mathrm{~mm}$ ).

Anverso: Busto diademado y drapeado de Juba I hacia la derecha y con cetro sobre el hombro; alrededor la leyenda REX IVBA.

Reverso: Templo octástilo y a sus lados leyenda neopúnica YWB'Y HMMLKT. Glóbulo central en lado central del templo.

Procedencia: Bertolami Fine Arts 24 (2016), lote 559.

2. Denario de Juba I (Mazard 1955: no 85; Alexandropoulos 2007: no 29A) (4.18 g; $20 \mathrm{~mm}$ ).

Anverso: Busto diademado y drapeado de Juba I hacia la derecha y con cetro sobre el hombro; alrededor la leyenda REX IVBA.

Reverso: Templo octástilo y a sus lados leyenda neopúnica YWB'Y HMMLKT. Sin glóbulo central en lado central del templo.

Procedencia: CNG 84 (2015), lote 761. 
3. Quinario de Juba I (Mazard 1955: no 87; Alexandropoulos 2007: no 30) (1.73 g; $14 \mathrm{~mm})$.

Anverso: Busto de una victoria hacia la derecha; alrededor la leyenda REX IVBA.

Reverso: Caballa a galope hacia la derecha bajo leyenda neopúnica YWB'Y HMMLKT. Procedencia: CNG Triton XVIII (2015), lot 798.

\section{TALLER DE CiRTA}

4. Quinario de Juba I (Mazard 1955: no 88; Alexandropoulos 2007: no 31) (1.81 g; $12 \mathrm{~mm})$.

Anverso: Busto diademado y drapeado de Juba I hacia la derecha y con cetro sobre el hombro.

Reverso: Caballa a galope hacia la derecha.

Procedencia: Mazard 1955: 241, no 88.

5. Sestercio de Juba I (Mazard 1955: no 89; Alexandropoulos 2007: no 32) (0.80 g; $6 \mathrm{~mm})$.

Anverso: Busto de África hacia la derecha y tocada con piel de elefante, con jabalina sobre su hombro.

Reverso: León caminando hacia la derecha y cabeza de frente; encima S invertida. Procedencia: Mazard 1955: 241, no 89.

6. Quinto o sexto de Juba I (Mazard 1955: no 90; Alexandropoulos 2007: no 33) (52.20 g; $37 \mathrm{~mm}$ ).

Anverso: Busto de Amón con cuernos y hacia la derecha.

Reverso: Elefante hacia la derecha bajo leyenda neopúnica SYWB'Y HMMLKT.

Procedencia: Mazard 1955: 241, no 90.

7. Tercio o cuarto de Juba I (Mazard 1955: no 91; Alexandropoulos 2007: no 34) (46.40 g; $35 \mathrm{~mm}$ ).

Anverso: Templo octástilo.

Reverso: Edificio de cinco columnas de las cuales tres son atlantes, sosteniendo un arquitrabe coronado; alrededor leyenda neopúnica SYWB'Y HMMLKT.

Procedencia: Mazard 1955: 241, no 91.

8. Unidad de Juba I (Mazard 1955: no 92; Alexandropoulos 2007: no 35) (11.31 g; $28 \mathrm{~mm})$.

Anverso: Busto de Amón con cuernos y hacia la derecha.

Reverso: Elefante hacia la derecha bajo leyenda neopúnica SYWB'Y HMMLKT.

Procedencia: Paul-Francis Jacquier 37 (2012), lote 199. 
9. Media unidad de Juba I (Mazard 1955: no 93; Alexandropoulos 2007: no 36) (7.36 g; $22 \mathrm{~mm}$ ).

Anverso: Busto de África hacia la derecha y tocada con piel de elefante.

Reverso: León hacia la derecha y cabeza de frente; encima leyenda neopúnica SYWB'Y HMMLKT.

Procedencia: Münzen \& Medaillen GmbH 44 (2016), lote 228.

\section{REFERENCIAS BIBLIOGRÁFICAS}

Alexandropoulos, J. (2000). La «Romanisation» des monnayages antiques de I'Afrique du Nord Orientale: analyse de quelques jalons. En M. P. García-Bellido y L. Callegarin (coords.), Los cartagineses y la monetización del Mediterráneo occidental (pp. 43-52). Madrid.

Alexandropoulos, J. (2005). Monnaie et romanisation en Afrique antique ler siècle av. J.-C.-Ile siècle ap. J.-C. Pallas, 68, pp. 203-216.

Alexandropoulos, J. (2007). Les monnaies de l'Afrique antique (400 av. J.-C.-40 ap. J.-C.). Toulouse.

Alexandropoulos, J. (2012). Aspects militaires de l'iconographie monétaire numide. CEA, 49, pp. 211-234.

Amela Valverde, L. (2000). Numidia y la clientela pompeyana. La acción de los políticos de la República romana en el extranjero. Iberia, 3, pp. 253-264.

Amela Valverde, L. (2001). El áureo de Cn. Pompeyo Magno (RRC 402), acuñado en Amisos (Ponto). GN, 140, pp. 5-13.

Amela Valverde, L. (2002). Las clientelas de Cneo Pompeyo Magno en Hispania. Barcelona.

Amela Valverde, L. (2010). El áureo de Cn. Pompeyo Magno (RRC 402/1). ETF(Hist), 23, pp. 205-216.

Amela Valverde, L. (2013). Salida a subasta de un áureo RRC 402/1, de Cneo Pompeyo Magno. En Varia Nummorum II, Barcelona, pp. 11-14.

Amisano, G. (2014). La storia di Roma antica e le sue monete. III. Gli anni delle guerre civil. Cassino. 
Bertrandy, F. (1976-1978). Remarques sur l'origine romaine du monnayage en bronze et en argent de Juba ler, roi de Numidie. $B C T H(B), 12 / 14$, pp. 9-22.

Burnett, A. (1987). Africa. En A. Burnett y M. H. Crawford (eds.), The Coinage of the Roman World in the Late Republic (pp. 175-185). Oxford.

Burnett, A., Amandry, M. y Ripollès, P. P. (1992). Roman Provincial Coinage. Volume I. From the death of Caesar to the death of Vitellius (44 B.C.-A.D. 69). Part I: Introduction and Catalogue. Londres/París.

Campana, A. (2001). Monete d'oro della repubblica romana VIII - Emissioni di Gneo Pompeo Magno (71 a.C.). PN, 148, pp. 20-24.

Camps, G. (1981). Les derniers rois numides, Massinissa II et Arabion. $B C T H(B), 17$, pp. 303-311.

Chaves Tristán, F. (2005). Guerra y moneda en la Hispania del Bellum Civile. En J. F. Rodríguez Neila et al. (coords.), Julio César y Corduba: tiempo y espacio en la campaña de Munda (49-45 a.C.) (pp. 207-245). Córdoba.

Coltelloni-Trannoy, M. (1999). Les représentations de l'Africa dans les monnayages africains et romains à l'époque républicaine. En Numismatique, langues, écritures et arts du livre, spécificité des arts figurés. Actes VIle Colloque international sur l'histoire et l'archéologie de l'Afrique du Nord (pp. 67-92). París.

Coltelloni-Trannoy, M. (2003). Juba. En Encyclopèdie Berbére. XXV. IseqqemârenJuba (pp. 3914-3938). Aix-en-Provenc.

Cowan, R. W. (2006). The Land of King Mane. A Pun at Horace, Odes 1.22.15. CQ, 56(1), pp. 322-324.

Cowan, R. W. (2008). Caesar Beats the Odds: The Battle of Ruspina. Ancient Warfare, 2(4), pp. 24-29.

Crawford, M. H. (1974). Roman Republican Coinage. Cambridge.

Crawford, M. H. (1985). Coinage and Money under the Roman Republic. Italy and the Mediterranean Economy. Londres. 
DT = Delestrée, L.-P. y Tache. M. (2002). Nouvel Atlas des Monnaies Gauloises, I. De la Seine au Rhin. Saint-Germain-en Laye.

Estarán Tolosa, M. J. (2012). Bilingüismo en las leyendas monetales: una peculiaridad de la numismática hispana y africana. Antesteria, 1, pp. 349-357.

Estarán Tolosa, M. J. (2016). Epigrafía bilingüe del Occidente romano. El latín y las lenguas locales en las inscripciones bilingües y mixtas. Zaragoza.

Falbe, C. T., Lindberg, J. Chr. y Müller, L. (1862). Numismatique de l'Ancienne Afrique. III. Les monnaies de la Numidie et de la Mauritanie, Copenhague.

Falomir Pastor, C. (2013). Juba II. Rey de los mauros y los libios. Valencia. URL: <http://roderic.uv.es/handle/10550/28428> (26/09/2018).

Fischer, B. (1978). Les monnaies antiques d'Afrique du Nord trouvées en Gaule. París.

Gozalbes Cravioto, E. (2016). La crisis de la República en el África romana: la actuación de César en 46 a.C. En G. Bravo y R. González Salinero (coords.), Crisis en Roma y soluciones desde el poder. Actas del XIII Coloquio de la Asociación Interdisciplinar de Estudios Romanos (pp. 65-82). Madrid/Salamanca.

Gsell, S. (1927). Histoire ancienne de l'Afrique du Nord. V. Les royaumes indigènes. Organisation sociale, politique et économique. París.

Günther, Sv. (2018). MFRP 23: Juba I. an Hunsrück: Zu zwei numidischen Denarfunden in der Gemarkung Mittelstrimming (Kries Cochem-Zell). NNB, 67(1), pp. 5-9.

Hill, G. F. (1903). Coins of Ancient Sicily. Westminster.

Hinard, F. (1989). Marius, Sylla et l'Afrique. En L'Africa romana. Atti del VI Convegno di studio (pp. 81-88). Sácer.

Kopij, K. (2016). The Context and Dating of the Pompey's Aureus (RRC 402). NAC, 45, pp. $109-127$.

Kormikiari, M. C. N. (2005). Numidian Royal Portraits. En XIII Congreso Internacional de Numismática Madrid - 2003. Actas - Proceedings - Actes I (pp. 349-356). Madrid. 
Kormikiari, M. C. N. (2007). Norte da África na antiguidade: os reis berberes númidas e suas iconografias monetarias. Revista do Museu de Arqueologia e Etnologia, São Paulo, 17, pp. 251-292.

Kormikiari, M. C. N. (2014). Espaços de poder no Norte da África berbere sob domínio cartaginês e romano: o caso de Cirta (Constantina, Argélia). Romanitas, 3, pp. 16-46.

Lacey, W. K. (1961). The tribunate of Curio. Historia, 10, pp. 318-329.

Lanzani, C. (1993). Silla e Pompeo. La spedizione di Sicilia e d’Africa. Historia, 7, pp. 343-362.

La Penna, A. (2000). La campagna di Curione in Africa. La narrazionee l'interpretazione di Cesare. En L'ultimo Cesare. Scritti Riforme Progetti Poteri Congiure (pp. 175210). Roma.

Le Bohec, Y. (2004). L'expédition de Curion en Afrique : étude d'histoire militaire. En L'Africa romana. Atti del XV convegno di studio. Ai confini dell'Impero: contatti, scambi, conflitti. Volume terzo (pp. 1603-1615). Roma.

Loscheider, R. (1997). Deux monnayages indigènes à la lumière du Bellum Civile entre César et Pompée : la série trévire en argent au nom d'ARDA et son prototype numide de Juba ler. CahN, 134, pp. 23-36.

MAA = Alexandropoulos, J. (2007). Les monnaies de l'Afrique antique (400 av. J.-C.40 ap. J.-C.). Toulouse.

Madjoub, M. (1998). Pompéius Magnus et les rois Maures. en L'Africa romana. Atti del XII Convegno di studio, III, Sácer, pp. 1321-1328.

Maritz, J. A. (2001). The Image of Africa: the evidence of the coinage. AClass, 44, pp. 105-125.

Marshall, B. (2016). An Aureus of Pompeius Magnus. Antichthon, 50, pp. 107-133.

Mazard, J. (1955). Corpus Nummorum Numidiae Mauretaniaque. París.

McDermott, W. C. (1969). M. Petreius and Juba. Latomus, 28, pp. 858-862. 
McDonnell-Staff, P. (2018). Final Fight with Elephants. The Battle of Thapsus, 46 BC. Ancient Warfare, 9(5), pp. 28-37.

Mesiano, F. (2011). A proposito dell’aureo di Pompeo. AlIN, 57, pp. 93-113.

Mirnik, I. (1987). Circulation of North African etc. Currency in Illyricum. Arheološki Vestnik, 38, pp. 369-391.

Offermann, H. (1977). Curio: Miles Caesaris? (Caesars Urteil über Curio in BC 2, 42). Hermes, 105, pp. 351-368.

Ott, F.-Th. (2013). Die Curio-Episode. En Die zweite Philippica als Flugschrift in der spâten Republik (pp. 344-415). Berlín/Boston.

Pera, M. (1998). Monete di Numidia nelle Collezioni di Palazzo Rosso a Genova. En L'Africa romana. Atti del XII Convegno di studio, III (pp. 1329-1334). Sácer.

Porte, F. (2017). César et la 'surprise strategique'. Logistique et guerre hivernale lors des campagnes de César en Epire et en Afrique (48-46 av. J.-C.). Aquila Legionis, 20, pp. 61-81.

Potz, E. (1998). Appians Klio dichtet. Die Curio-Episode bei Appian (E 2, 44, 175-45, 185) und Caesar (b.c. II 23-44). Philologus, 142, pp. 293-299.

Ranucci, S. (2007). Un ripostiglio di monete romane da Foligno. AlIN, 53, pp. 115153.

Roller, D. (2003). The World of Juba II and Kleopatra Selene. Londres.

$R P C \mathrm{I}=$ Burnett, A., Amandry, M. y Ripollès, P. P. (1992). Roman Provincial Coinage. Volume I. From the death of Caesar to the death of Vitellius (44 B.C.-A.D. 69). Part I: Introduction and Catalogue. Londres/París.

$R R C=$ M. H. Crawford, Roman Republican Coinage. Cambridge.

$R R C H=$ M. H. Crawford, Roman Republican Coin Hoards. London.

Salcedo Garcés, F. (1996). África. Iconografía de una provincia romana. Madrid.

Saylor, C. (1982). Curio and Antaeus: The African Episode in Lucan Pharsalia IV. TAPhA, 112, pp. 169-177. 
Sear, D. R. (1998). The History and Coinage of the Roman Imperatores 49-27 BC. Londres.

Sidiropoulos, K. (2011). A Hoard of denarii and Early Roman Messene. En Proceedings of the 14th International Numismatic Congress. Glasgow 2009. Vol. 1 (pp. 10251036). Glasgow.

Trell, B. L. (1976). Architecture on Ancient Coins. Archaeology, 29, pp. 6-13.

Trell, B. L. (1988). Ancient Coins as Evidence for the History of Art. Bulletin of the Asia Institute, 2, pp. 53-65.

Walthall, D. A. (2013). A hoard containing Late Republican 'denarii' from Morgantina (Sicily). AJN, 25, pp. 171-178.

Woytek, B. (2003). Arma et Nummi. Forschungen zur römischen Finanzgeschichte und Münzprägung der Jahre 49 bis 42 v. Chr. Viena.

Woytek, B. (2015). The Aureus of Pompey the Great Revisited. En P. van Alfen et al. (eds.), Fides. Contributions to Numismatics in Honor of Richard B. Witschonke (pp. 403-426). Nueva York.

Xhyheri, S. y Muçaj, Sk. (2014). A Coin Juba I of Numidia from Byllis and the Tropaeum of Cicero the Younger. Candavia, 4, pp. 83-93 [en albanés]. 